\title{
Surgery of less common conditions in leprosy
}

\author{
SANJAY SANE \\ Bandorawalla Leprosy Hospital and Diabetic Unit, K.E.M. Hospital \\ Pune, 55 Mahesh Society, opposite Bibbewadi Police Station, Pune, \\ India 411037
}

\section{Introduction}

The uncommon conditions in leprosy requiring surgical correction are either the direct result of the disease or secondary to nerve paralysis. They usually occur in the later stages of leprosy and are uncommon today, thanks to early treatment of leprosy by multidrug therapy (MDT). However, in areas where treatment has only started recently, there is a backlog of such deformities needing correction. These conditions are:

Primary deformities: these are directly due to the disease.

1. On the face: megalobules, nasal perforation, depression of nose, and loss of eyebrows.

2. In the extremities: contractures of fingers and toes.

Secondary deformities: these are usually secondary to trunk nerve paralysis.

1. Lagopthalmos.

2. Facial paralysis.

3. Wrist-drop.

4. Triple paralysis of the hand.

5. Neuro-arthropathies (e.g. carpal disintegration in the wrist and tarsal disintegration in the foot).

6. Gynaecomastia is secondary to involvement of the testis.

All these are disabling conditions that may not occur if early treatment of leprosy is instituted and many of them can be reversed by proper treatment. Early and effective institution of MDT is the best way of prevention of these deformities.

\section{How are these caused?}

PRIMARY DEFORMITIES

These occur by direct involvement of the soft tissues, particularly the skin and the mucosa, by

Correspondence to: S. Sane, Diabetic Unit, K.E.M. Hospital Pune, 55 Mahesh Society, opposite Bibbewadi Police Station, Pune, India 411037 
leprosy granuloma. Resolution of the granuloma in the skin leads to loss of collagen substrate. The skin becomes lax, causing facial wrinkles and elongation of the ear lobules. The integumental structures such as hair follicles and sweat glands are also destroyed in this process. Loss of eyebrows is one result. Perforation of the nose and depression of nose is due to the loss of the inner lining, loss of the nasal cartilage and secondary infection. Direct involvement of the skin and later of other soft tissues in reactional states of lepromatous leprosy results in severe contractures of the fingers and toes.

\section{SECONDARY DEFORMITIES}

These are secondary to involvement of a specific organ or area. Lepromatous infiltration of the testis causes testicular atrophy. The resulting excess of female hormones may lead to gynaecomastia, i.e. enlargement of the breast seen in male leprosy patients. Uncommon trunk nerve involvement, e.g. of the facial nerve outside the skull, of the radial nerve in the arm or forearm, median nerve near the elbow, or of all the three nerves innervating the hand, leads to the corresponding paralytic deformities. Involvement of the nerves supplying the joints of the hand or the foot leads to neurotrophic arthropathies.

\section{Prevention}

Prevention is by early treatment of leprosy and appropriate action in the early stages of the deformity. Treatment must begin before the soft tissue changes set in. During reactions, the affected parts should be kept clean, aseptic and at rest. The nose is kept clean by saline washes and this also prevents secondary infection. The affected hands, fingers and feet and toes are appropriately splinted in their correct positions, to prevent contractures and external injury. Anti-inflammatory and immunosuppressive drugs and drugs providing symptomatic relief can prevent many of these deformities.

Though these conditions are uncommon, for an individual patient they are of immense importance, being disabling and ugly. Thus, every medical or paramedical person working in leprosy should know of these conditions, that these can be treated surgically and where and when to refer them for surgery. With the exception of lagopthalmos, which if not urgently treated may lead to damage to the eye and even blindness, all other conditions are surgically treated after the reactions subside and the disease is well controlled.

Early diagnosis and MDT has reduced the occurrence of deformities, especially in the lepromatous spectrum.

Deformities can occur:

1. Following resolution of the lepromatous granulomata.

2. Due to loss of hair follicles and laxity of skin resulting from loss of elastic and collagen tissue.

3. Secondary to involvement of the nerves.

4. Due to the loss of testicular function following leprous involvement.

Though these deformities are uncommon, for the patient they are a cause of social isolation and their surgical correction is of immense importance. 


\section{Surgical treatment}

The treatment of all these conditions is through well-established methods of plastic and reconstructive surgery.

\section{Surgery for the depressed nose}

1 Before the development of the saddle nose, there is a period when infiltration of nasal mucosa by Mycobacterium leprae leads to nasal stuffiness, congestion and discharge. As the disease progresses, there is ulceration of the inner nasal lining that may lead to perforation or external nasal fistula.

2 The cartilaginous part of the nasal septum receives its nourishment from this lining. Repeated nasal ulceration leads to destruction of the septal cartilage and ultimate collapse of the nasal bridge, leading to the deformity. The loss of nasal spine of the maxilla inferiorly also contributes to the deformity. The process of ulceration can be arrested in the earlier stages by health care and medication. Established deformity can be effectively corrected by reconstructive surgery.

3 The causation of the deformity can be easily understood if the nose is likened to a single pole tent with the nasal septum as the central support and skin and the mucoperiosteum as outer and inner linings, respectively.

Surgical reconstruction of the nose requires understanding of three important factors in the causation of the deformity.

1. Except in those cases of external nasal fistula, there is no loss of the external skin of the nose; it simply telescopes in and becomes tethered to the underlying bone.

2. There is loss of internal lining of the nose (mucoperiosteum) due to repeated ulceration, leading to contracture and scarring.

3. There is loss of cartilaginous central nasal support. It must be reconstructed with the help of a bone graft or a synthetic support. Thus in any reconstruction, dissection of the tethered skin, providing adequate internal lining of skin and providing internal support are important prerequisites for a good result (Figure 1).

Development of saddle nose is a slow process and is totally preventable in the early stages. Integrity of the nose depends on the cartilaginous central support and the inner lining and outer skin. If either is damaged, the nose is deformed.

Reconstruction is based on:

1 Providing a central support, usually by a bone graft or a prosthesis and,

2 Restoring the internal lining by skin grafting.

The post-nasal inlay operation provides complete nasal cosmetic restoration.

\section{Surgery for lagopthalmos}

The condition is due to involvement of the facial nerve and subsequent paralysis of the muscles supplying the eyelids. The usual signs and symptoms are: 

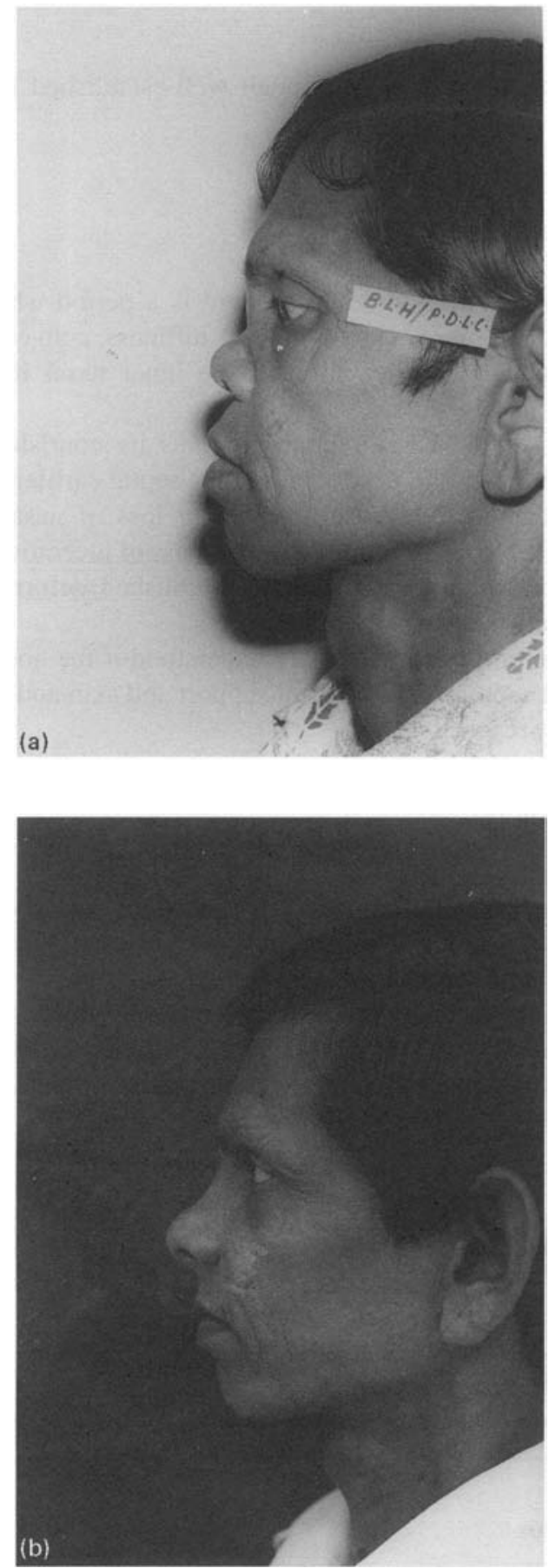

Figure 1. Facial appearance (a) before and (b) after correction of nasal deformity. 
1. Inability to close the eye fully voluntarily; there is an appreciable gap on tight voluntary closure.

2. The eye remains half-open during sleep.

3. There is watering and dryness of the eyes.

4. The eye looks bigger than the other eye

Prevention is by detecting early onset of paralysis of the ocular muscles. The patient complains of excessive watering of the eyes and notices a sluggish response of eyelid closure to any external stimulus. Appropriate measures instituted early can prevent this deformity. These consist of medication to reduce the nerve inflammation and oedema, supportive vitamins for nerve recovery, physical exercises and electrical stimulation. Dark glasses are given to protect the eyes from exposure keratitis and subsequent blindness and tear substitutes to prevent dryness.

\section{INDICATIONS FOR SURGERY}

After 6 months, if attempts at treatment fail, surgical intervention is mandatory. In an established case, absence of any protective measures to protect the anaesthetic eye warrants surgery on an emergency basis.

The common procedures are tarsorrhaphy and temporalis transfer.

Passive permanent reduction of palpebral fissure by medial or lateral tarsorraphy is one solution. This is a static procedure, a simple operation that can be learnt by an inexperienced surgeon.

Active eyelid closure with the help of a temporalis muscle sling operation is a dynamic procedure. The Gillie's operation and its Johnson's variation are the two common methods. In the latter, instead of temporalis fascia, a free graft of fascia lata or palmaris longus is used to extend a partially detached tendon of the temporalis muscle. Postoperative care, especially only soft food for 3 weeks and training for integration of the transfer, is essential for achieving a good result (Figure 2).

Paralysis of the eyelids is a slow process and responds very well to the conservative line of treatment, if diagnosed early.

Surgical correction of lagopthalmos is mandatory to protect the eye from danger of repeated keratitis and consequent total blindness.

A simple static procedure like tarsorraphy can be done by any qualified eye surgeon. The temporalis sling operation, a dynamic procedure for the closure of the eyelids, requires a trained reconstructive surgeon.

\section{Loss of eyebrows, sagging of facial skin and megalolobule}

\section{SURGICAL CORRECTION OF LOSS OF EYEBROWS}

This is done by using one of the following three methods: a direct free graft from behind the ear or scalp, a temporal artery flap, and direct hair implantation, e.g. by multiple punch grafts. All methods can yield excellent result. Care must be taken regarding the patient's preference and aesthetics (Figure 3). 

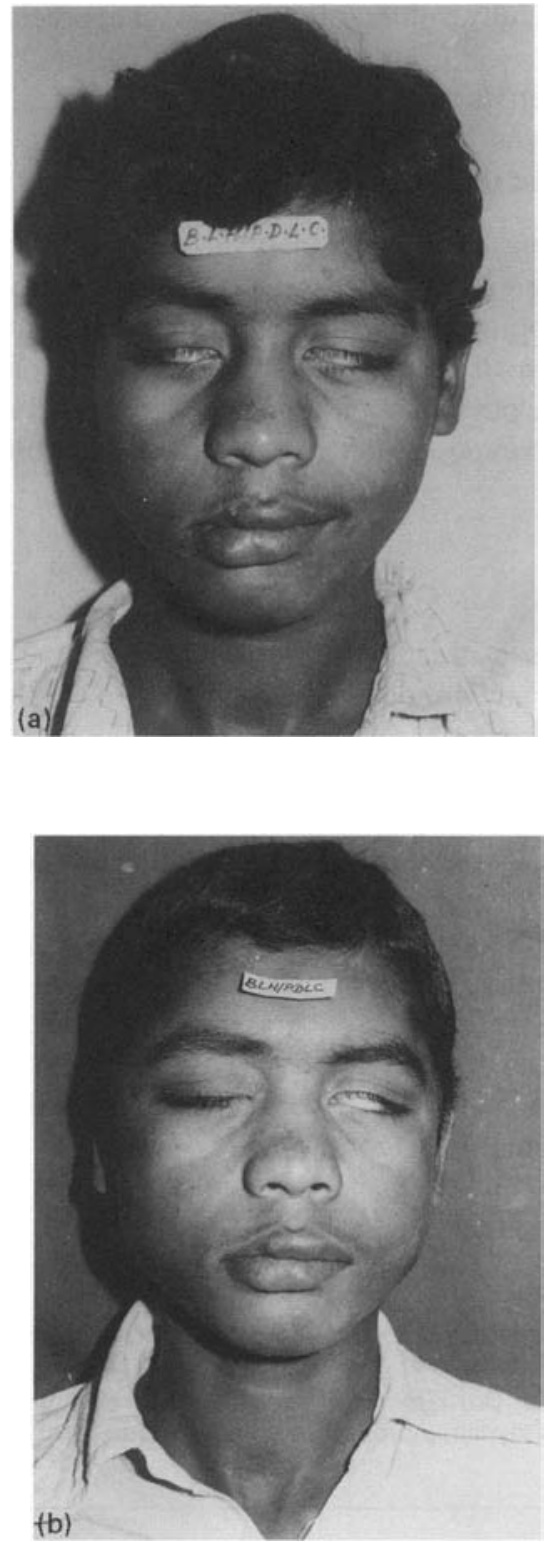

Figure 2. (a) Pre- and (b) postoperative appearance after a temporalis transfer operation using the Johnson method.

FACE-LIFT OPERATIONS

The facial skin is sometimes affected in lepromatous leprosy by the disease process. Atrophy of tissues subsequent to granulomatous infiltration causes sagging of skin. Though the deformity does not cause any functional loss, the patient's appearance prevents them being 

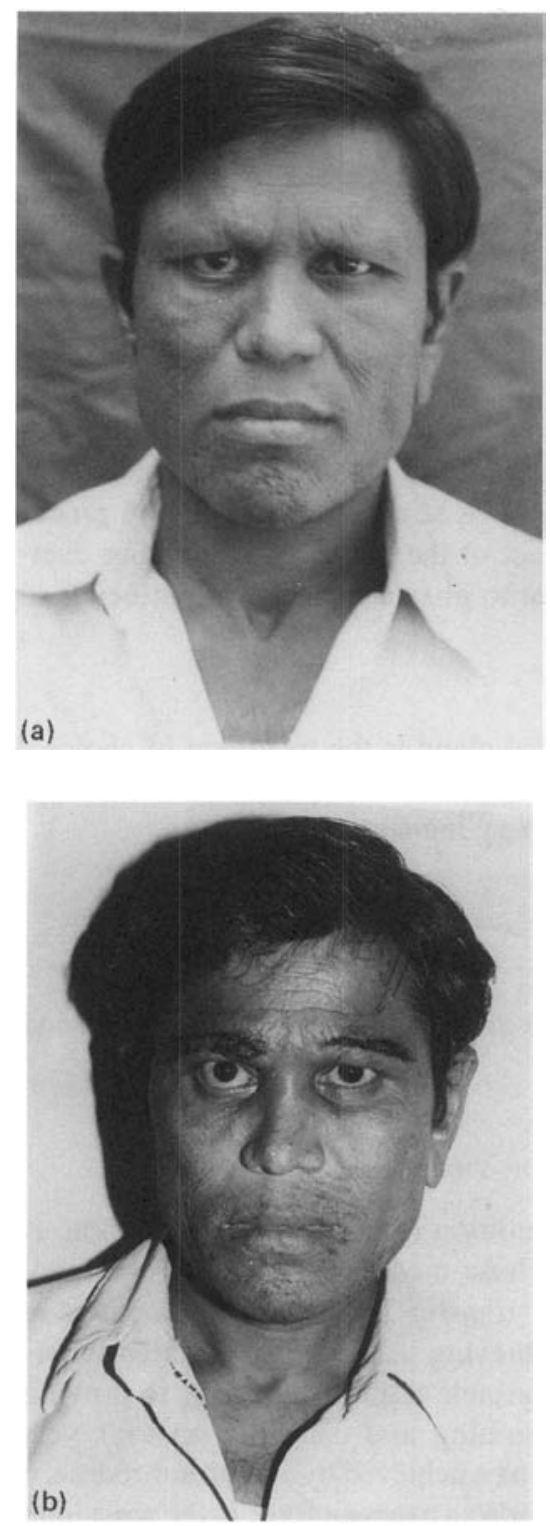

Figure 3. Surgical correction of loss of eyebrows. (a) Before and (b) after surgery.

accepted in society. Thus facial reconstruction is of prime importance and is the first step in the rehabilitation process in these patients.

When the disease is active, granulomatous infiltration causes distension of the skin and destruction of the collagen infrastructure, which is responsible for the toughness of the skin and its elasticity. Resolution of the granulomata with effective drug therapy leaves loose sagging skin without collagen support, and the patient's facial skin is furrowed with lax skin 
folds. Thus there is redundant facial skin mainly around the nose, chin and in the upper neck, and also in the posterior part of the face. Surgery aims at excision of the redundant skin and re-tensioning the skin so as to remove the wrinkling. This is done through careful skin incisions, which are hidden and planned in the hairline, and great care is taken to preserve and not to damage the delicate facial nerve branches supplying the facial muscles. When there is total involvement of skin, a classical face-lift operation is required. More commonly, anterior or circumoral face-lift is sufficient.

\section{MEGALOLOBULE}

The process that disfigures the face is responsible for the deformity of the ears. The skin of the pinna and the lobule becomes lax. Excision of the redundant skin and trimming followed by refashioning is required and is a straightforward simple procedure. The skin incisions are placed on the posterior aspect of the pinna, thus avoiding over- or under-correction. These operations enable the patient to mix in society without being stigmatized.

\section{GYNAECOMASTIA}

Excision of the hypertrophied gland is the treatment of choice. This can be done in smaller glands by a circumareolar cosmetic incision, which leaves little scar. Larger glands are excised through a submammary incision.

\section{Nerve lesions}

Appropriate tendon transfers are used to treat the paralytic conditions. Only the triple nerve paralysis is described here.

\section{TRIPLE NERVE PARALYSIS OF THE HAND}

This extremely disabling condition is fortunately uncommon. Almost all the muscles in the forearm are affected. Only those muscles supplied by the median nerve in the forearm are spared, and can be used for transfer. With very few options remaining for reconstruction, priority is now more on achieving a functionally stable wrist first, then to provide finger extension, to provide an opposable thumb and lastly, to provide claw finger correction. The whole process is time consuming and can take up to 1 year, but the results are fairly satisfactory. Wrist stability can be achieved by a wrist arthrodesis in 15-20 degrees dorsiflexion, or by a tendon transfer to provide an extensor force at the wrist joint, usually done by transferring the pronator teres muscle. Reconstitution of finger extensors is done by transferring flexor carpi radialis. Opponens replacement is accomplished by transferring the ring finger superficial to the short abductor insertion on the thumb. Claw finger correction is done either by a superficial flexor transfer or by capsulorraphy of the maetacarpo-phalangeal joints.

\section{Neuroarthopathy}

Involvement of the nerve supply of joints together with minor injuries causes this condition and is more common in the feet, involving the mid-tarsal joints. The treatment is by rest and 
the use of a special orthotic device as such as patellar tendon bearing orthosis, which must be used for a long period before stabilization occurs. In some cases, operations for correction of deformity and stabilization are required. These must be followed by appropriate orthotic devices to prevent direct weight bearing on these joints.

\section{Claw toes}

This condition, though not uncommon, is commonly neglected and hence is mentioned here. Claw toes are due to paralysis of the intrinsic muscles of the foot. This is the result of the involvement of the posterior tibial nerve or its branches and causes the hyperextension of the toes at the metacarpophalangeal joint and flexion at the interphalangeal joint. This leads to excessive pressures on the tips of the toes while walking and metacarpophalangeal joint hyperextension, which exposes the metatarsal head to the shearing forces leading to ulceration. Its treatment can significantly reduce the incidence of plantar ulceration under the metatarsal heads. Correction is by transfer of the toe flexors to the extensors. In this operation, the flexor digitorum longus tendon of each toe is detached from its insertion, brought out onto the dorsum of the toe, and sutured to the extensor expansion. The foot is immobilized for 3 weeks, after which the patient is prescribed MCR footwear.

It should be emphasized again that all these conditions, though uncommon, are still found even in areas where leprosy has a low prevalence. For the patient, the correction of these deformities is of major importance. Patients should have access to facilities where these corrections can be done.

\section{Acknowledgement}

The photographs in this article are of patients operated on in the Dr Bandorawalla Leprosy Hospital, Khondwa, Pune, India. 